\section{Standard Error of the Mean}

Michael Franzen

Allegheny General Hospital, Pittsburgh, PA, USA

\section{Definition}

If a test is given to a certain sample, a mean score can be calculated. If we then give the test to a group of samples and calculate mean scores for each sample, the standard error of the mean can be thought of as the standard deviation of the mean scores in all of the samples. The population mean can never be precisely known. The standard error of the mean gives some indication of the variability possible in various samples drawn from that population. As with using samples to estimate population values, the larger the sample, the more likely that the mean of that sample would approximate the population mean, and the smaller would be the standard error of the mean.

In clinical neuropsychology, as in all of psychological measurements, test scores are never perfectly reliable. There is always some unreliability. Many times in clinical neuropsychology, there may be multiple publications of mean scores drawn from different samples. For example, the Rey Auditory Verbal Learning Test has published "normative" data from older samples, VA samples, outpatient samples, and inpatient psychiatric samples, among others. These samples vary in size, and frequently the mean scores and standard deviations vary across the samples as well. Being able to calculate the standard error of the mean allows us to estimate how different these sample means are in relation to the population mean and thereby tell us how confident we can be in comparing our obtained scores to the mean scores.

\section{References and Readings}

Nunnally, J., \& Bernstein, I. (1994). Psychometric theory (3rd ed.). New York: McGraw Hill. 